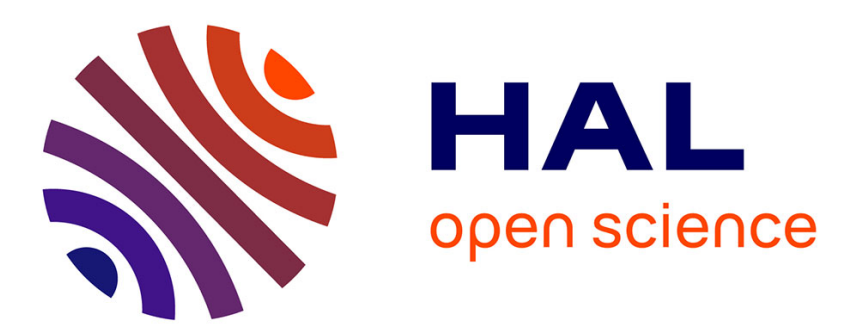

\title{
Inverse conduction and advection in a flat channel with transient external thermal excitation and observation
}

Waseem Al Hadad, Denis Maillet, Yves Jannot, Vincent Schick

\section{To cite this version:}

Waseem Al Hadad, Denis Maillet, Yves Jannot, Vincent Schick. Inverse conduction and advection in a flat channel with transient external thermal excitation and observation. International Journal of Heat and Mass Transfer, 2018, 127, pp.362-372. 10.1016/j.ijheatmasstransfer.2018.05.142 . hal-01962419

\author{
HAL Id: hal-01962419 \\ https://hal.science/hal-01962419
}

Submitted on 20 Dec 2018

HAL is a multi-disciplinary open access archive for the deposit and dissemination of scientific research documents, whether they are published or not. The documents may come from teaching and research institutions in France or abroad, or from public or private research centers.
L'archive ouverte pluridisciplinaire HAL, est destinée au dépôt et à la diffusion de documents scientifiques de niveau recherche, publiés ou non, émanant des établissements d'enseignement et de recherche français ou étrangers, des laboratoires publics ou privés. 


\title{
Inverse conduction and advection in a flat channel with transient external thermal excitation and observation
}

\author{
Waseem Al Hadad ${ }^{\mathrm{a}, \mathrm{b}, *}$, Denis Maillet ${ }^{\mathrm{a}, \mathrm{b}}$, Yves Jannot ${ }^{\mathrm{a}, \mathrm{b}}$, Vincent Schick $^{\mathrm{a}, \mathrm{b}}$ \\ ${ }^{a}$ Université de Lorraine, LEMTA(UMR 7563), ENSEM, 2 Avenue de la Forêt de Haye, \\ BP 90161, 54505 Vandouvre-lès-Nancy cedex, France \\ ${ }^{b}$ CNRS, LEMTA(UMR 7563), BP 90161, 54505 Vandœuvre-lès-Nancy cedex, France
}

\begin{abstract}
The transient profiles of temperature and normal heat flux inside a flat minichannel heated by a surface heat source are constructed from temperature measurement over its external heated face. It uses analytical expressions of the corresponding transfer functions which are calculated using Laplace and Fourier integral transforms. Firstly, this estimation technique is verified on synthetic outputs of a finite elements code (COMSOL). Then it is implemented on an experimental minifluidic bench with electrical heating and temperature measurement by thermocouples and infrared thermography, for a low Péclet number of the flow. The presented results show that the heat source can be recovered at any time, as well as the internal normal heat flux and temperature distributions, including the bulk temperature of the liquid flow.
\end{abstract}

Keywords: conduction and advection, conjugate heat transfer, minichannel, unsteady heat transfer, convolution and deconvolution, inverse problems, measurement and instrumentation.

\section{Nomenclature}

$\ell \quad$ channel length, $\mathrm{m}$

$\mathbb{H} \quad$ Heaviside function

*. Corresponding author. Tel. :(+33)3 83595585 ; fax :+33 383595551

Email address: waseem.al-hadad@univ-lorraine.fr (Waseem Al Hadad )

Preprint submitted to International Journal of Heat and Mass Transfer 8 juin 2018 
$\mathcal{H}$ transfer function

$\mathrm{P} \quad$ given point

$h$ heat transfer coefficient, $\mathrm{W} \cdot \mathrm{m}^{-2} \cdot \mathrm{K}^{-1}$

$L \quad$ virtual channel length, m

Pe Péclet number

Re Reynolds number

$T$ temperature, $\mathrm{K}$

$T_{\infty} \quad$ ambient temperature, $\mathrm{K}$

$V_{m} \quad$ mean velocity, $\mathrm{m} . \mathrm{s}^{-1}$

$x, y$ spatial coordinates, $\mathrm{m}$

$a \quad$ thermal diffusivity, $\mathrm{m}^{2} \cdot \mathrm{s}^{-1}$

$p \quad$ Laplace parameter, $\mathrm{s}^{-1}$

$u$ input, cause, excitation

$W \quad$ transmittance

$y \quad$ output, consequence, response

Z impedance

\section{Greek symbols}

$\alpha_{n} \quad$ discrete eigenvalue of order $n$

$\lambda \quad$ thermal conductivity, $\mathrm{W} \cdot \mathrm{m}^{-1} \cdot \mathrm{K}^{-1}$

$\nu \quad$ kinematic viscosity, $\mathrm{m}^{2} \cdot \mathrm{s}^{-1}$

$\Phi$ heat flow rate (surface integral of $\varphi$ ), W

$\rho \quad$ density, $\mathrm{kg} \cdot \mathrm{m}^{-3}$

$\varphi \quad$ heat flux density in $y$ direction, W. $\mathrm{m}^{-2}$

superscripts

- $\quad$ Laplace transform

Fourier transform 


$\begin{array}{ll}\text { in } & \text { inlet } \\ \text { out } & \text { outlet } \\ \text { ss } & \text { steady state } \\ T & \text { transposed of a matrix } \\ \text { subscripts } \\ f \quad \text { fluid } \\ s & \text { solid }\end{array}$

\section{Introduction}

In our previous works, we have shown the interest of transfer functions for linear time invariant systems where heat diffusion and advection occur, both on a theoretical [1] and on an experimental [2] basis. In this paper, which deals with a flat mini heat extractor, we will show how the surface heat source as well as the internal state variables (temperature and heat flux) can be estimated from temperature measurements over one of the outer faces using the correponding transfer function. This non-destructive estimation technique, that corresponds to the construction of a virtual sensor, allows us therefore to estimate, in steady and transient regimes, the thermal state at locations difficult to access using direct measurements at another easy to access location. This requires the system to be linear with a geometry, thermophysical properties and fluid velocities that do not vary with time.

So, the topic dealt with in this paper derives from the now classical Inverse Heat Conduction Problem (IHCP) introduced by J.V. Beck et al. in the 80's [3] which consists in reconstructing surface temperatures or heat fluxes at part of the boundary of a solid domain, using known boundary conditions over its complementary part, the missing information being replaced by internal temperature measurements. This type of problem is mathematically ill-posed because the presence of noise in the data tends to make reconstruction of temperature or flux at the unknown part of the boundary unstable. This requires some special class of data processing called "regularization". This IHCP approach is very useful to estimate experimentally the distributions of both heat flux and temperature over the inner surface of the heated wall of a channel, using the diffusion heat equation in its solid volume, in 
order to derive the profiles of the internal convection coefficients. It can be used, for example, to optimize internal fluid mechanics in such a channel, see [4].

In Inverse Forced Convection Problems (IFCP) [5], the problem at stake is exactly the same, but the studied domain is a flowing fluid whose velocity field is known. The first works about this type of problems appeared in the 1990's and concerned estimation of inlet space [6] or time [7] temperature distributions in a flat heated channel [6] or the wall heat flux estimation in a flat [5] [8] or annular [9] channel in transient thermal regime.

The precise subject of our work is the Inverse Conjugate Forced Convection Problem (ICFCP) : the type of heat equation to be inverted is still the forced convection heat equation but it concerns not only a fluid, but also a solid subdomain, where a zero velocity field prevails, with the specific character that no heat transfer coefficient is used at their interface anymore. Few papers can be found yet for ICFCP, see [10] for example, since articles on the corresponding direct problem only date back to the early 2000's, see [1] [12] [13] [14. However, to our knowledge, no work on ICFCP can be found with inversion not only of simulated measurements but also of real ones.

Our paper is organized as follows : in section 2, we introduce the studied system (here the heat extractor) and its modelization. In section 3 , we derive the methodology for estimating the internal conditions from measurements over one of the external faces as well as the corresponding transfer function in a mini-heat extractor. In section 4, we validate the methodology of sections 2 and 3 using synthetic profiles generated by COMSOL. In section 5 , we apply this proposed methodology to a real experiment and will show the corresponding results.

\section{The studied system and its transient modeling}

\subsection{The studied system}

Let us consider a laminar fluid flow in a channel of length $2 \ell$, of thickness $e_{f}$, limited by two parallel plates of polycarbonate of thicknesses $e_{1}$ and $e_{2}$, see Figure 1. The velocity profile $u(y)$ is assumed to be parabolic (Poiseuille flow) and fully developed from the inlet to the outlet of channel. The two solid 
layers (walls) and the fluid layer are characterized by their thermal conductivity $\lambda_{i}$, their volumetric heat $\rho c_{i}$ and their thermal diffusivity $a_{i}=\lambda_{i} / \rho c_{i}$ where $i=s_{1}, s_{2}$ or $f$ respectively.

A surface heat source, $q(x, t)$ is imposed between $x_{1}$ and $x_{2}$ on the lateral hot face noted here $h$. The two lateral faces (hot $h$ and cold $c$ ) exchange heat with its surrounding environment (here ambient air) which is at an unifrom temperature $T_{\infty}$. These exchanges are characterized by coefficients $h_{1}$ and $h_{2}$ respectively. These ones are assumed to be uniform on each face (they integrate natural convection and linearized radiation).

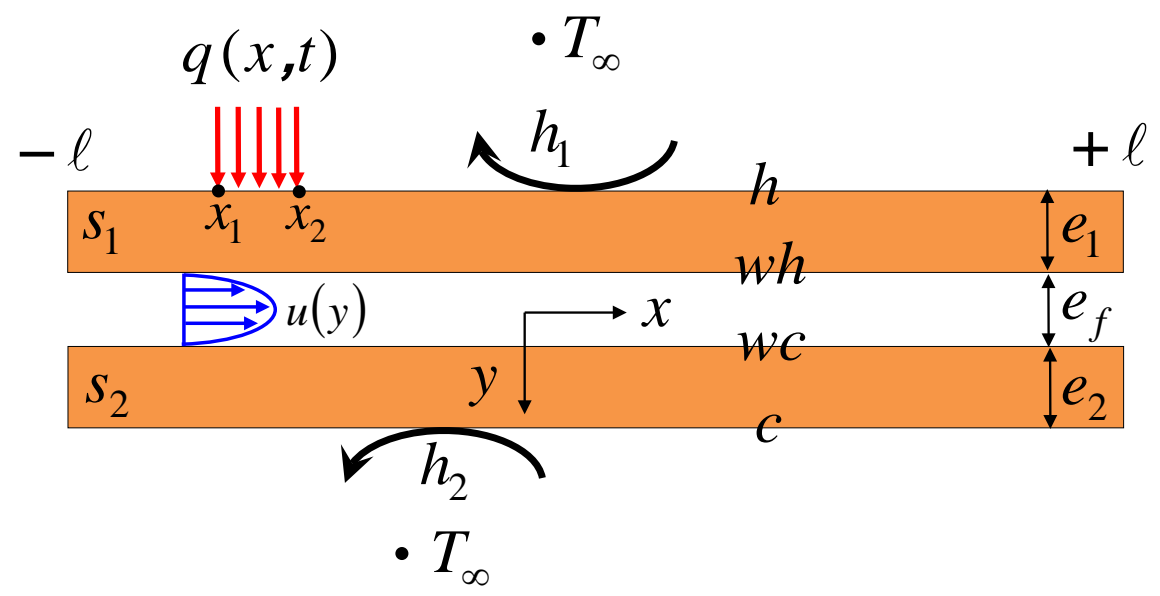

FiguRE 1: $2 D$ model of flow and heat transfer in the flat channel.

\subsection{Modelization}

The heat equation describing $2 D$ heat transfer in transient state in the walls (upper wall, $s_{1}$ and lower wall, $s_{2}$ ), in the fluid layer $(f)$ and the corresponding boundary and initial conditions, are :

- Heat equation in the solid (wall) :

$$
\frac{\partial^{2} T_{s_{i}}}{\partial x^{2}}+\frac{\partial^{2} T_{s_{i}}}{\partial y^{2}}=\frac{1}{a_{s_{i}}} \frac{\partial T_{s_{i}}}{\partial t} \quad \text { with } \quad i \equiv 1 \text { or } 2
$$


- Heat equation in the fluid :

$$
\frac{\partial^{2} T_{f}}{\partial x^{2}}+\frac{\partial^{2} T_{f}}{\partial y^{2}}-\frac{u(y)}{a_{f}} \frac{\partial T_{f}}{\partial x}=\frac{1}{a_{f}} \frac{\partial T_{f}}{\partial t}
$$

- External in-plane boundary conditions :

$$
\begin{gathered}
\varphi_{h}(x, t)=q(x, t)-h_{1}\left(T-T_{\infty}\right) \quad \text { at } y=-\frac{e_{f}}{2}-e_{1} \\
\varphi_{c}(x, t)=-h_{2}\left(T-T_{\infty}\right) \quad \text { at } y=+\frac{e_{f}}{2}+e_{2}
\end{gathered}
$$

where $\varphi_{h}(x, t)$ and $\varphi_{c}(c, t)$ are the heat fluxes in the $y$ direction, on the $h$ and $c$ faces respectively. $q$ is the surface density of the heat source power. We assume here that $q(x, t)$ is separable and can be written as the product of a transient intensity $Q(t)$ (in $W$ ) by a space distribution $f(x)\left(\right.$ in $\left.m^{-2}\right)$ :

$$
q(x, t)=Q(t) f(x)
$$

- The solid/fluid interface conditions : at the solid/fluid interfaces, we assume the continuity conditions of heat flux and temperature :

$$
-\lambda_{s_{i}} \frac{\partial T_{s_{i}}}{\partial y}=-\lambda_{f} \frac{\partial T_{f}}{\partial y} \quad \text { and } \quad T_{s_{i}}=T_{f} \quad \text { at } \quad y= \pm \frac{e_{f}}{2}
$$

where $i \equiv 1$ if $y=-e_{f} / 2$ and $i \equiv 2$ if $y=+e_{f} / 2$

- The axial boundary conditions are periodicity boundary conditions :

$$
\frac{\partial T_{i}}{\partial x}=0 \quad \text { and } \quad T_{i}=T_{\infty} \quad \text { at } \quad x= \pm L \quad \text { for } \quad i \equiv s_{1}, s_{2} \text { or } f
$$

Here length $2 L$, with $k_{v}=L / \ell>1$, corresponds to the virtual length of the channel, see [1].

- Initial condition :

$$
\left.T\right|_{x, y, t=0}=T_{\infty}
$$


The developed parabolic velocity field $u(y)$ within the flow can be made homogeneous through the parameterization of the fluid layer into $K$ fluid layers of thicknesses $e_{k}=y_{k}-y_{k-1}$, with $y_{0}=-e_{f} / 2$, see Figure 2. The exact velocity distribution $u(y)$ and its parameterized distribution $u_{k}$ can be written as :

$$
u(y)=\frac{3}{2} U_{m}\left(1-4\left(\frac{y}{e_{f}}\right)^{2}\right) \Rightarrow u_{k}=\frac{3}{2} U_{m}\left(1-\frac{4 K}{3 e_{f}^{3}}\left(y_{k}^{3}-y_{k-1}^{3}\right)\right)
$$

where $U_{m}$ is average velocity and $K$ is the number of fluid sublayers.

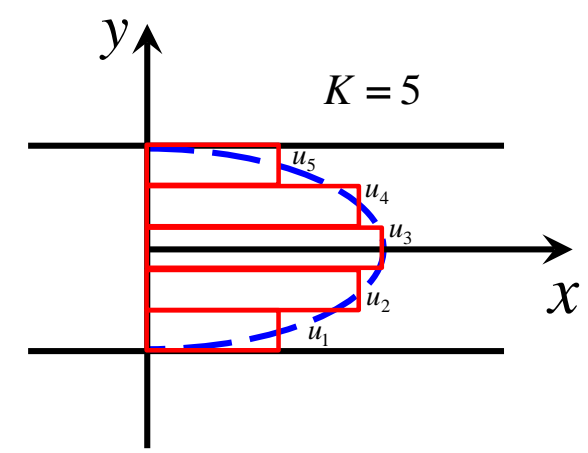

Figure 2: Parameterization of $u(y)$ into a piecewise constant function with $K=5$ parameters.

A change of function is made in order to define the temperature increase $\theta$, that is the response to the surface heat source $q(x, t)$ :

$$
\theta(x, y, t)=T(x, y, t)-T_{\infty}
$$

The Laplace transform $\bar{\theta}$ in time as well as the Fourier transform $\tilde{\theta}$ in axial direction of temperature increase $\theta$ for the $[-L ;+L]$ interval, are defined by :

$$
\begin{gathered}
\bar{\theta}(x, y, p)=\int_{0}^{\infty} \theta(x, y, t) e^{-p t} \mathrm{~d} t \\
\tilde{\theta}_{n}(y, t)=\int_{-L}^{+L} \theta(x, y, t) e^{-\iota \alpha_{n} x} \mathrm{~d} x \quad \text { with } \quad \iota^{2}=-1
\end{gathered}
$$


The discrete eigenvalues $\alpha_{n}=n \pi / L$ are defined here for any relative integer $n$. Fourier inversion of (12) requires a truncation to an even number $N_{h}=2 n_{h}$ of harmonics, where $n_{h}$ is the number of harmonics of strictly positive order :

$$
\theta(x, y, t) \equiv \frac{1}{2 L} \sum_{n=-\infty}^{\infty} \tilde{\theta}_{n}(y, t) e^{\iota \alpha_{n} x} \approx \frac{1}{2 L} \sum_{n=-n_{h}+1}^{n_{h}} \tilde{\theta}_{n}(y, t) e^{\iota \alpha_{n} x}
$$

After applying the Laplace and Fourier transformations to the solid and fluid heat equations (1) and (2) respectively, and by taking into account axial boundary conditions (7) as well as the initial condition (8), we get an ordinary differential equation in each domain :

$\triangleright$ In the walls :

$$
\frac{d^{2} \tilde{\bar{\theta}}_{s_{i_{n}}}}{d y^{2}}-\beta_{n}^{2} \tilde{\bar{\theta}}_{s_{i_{n}}}=0 \quad \text { with } \quad \beta_{n}^{2}=\left(\alpha_{n}^{2}+\frac{p}{a_{s_{i}}}\right)
$$

$\triangleright$ In each fluid layer :

$$
\frac{d^{2} \tilde{\bar{\theta}}_{f_{n}}}{d y^{2}}-\gamma_{k}^{2} \tilde{\bar{\theta}}_{f_{n}}=0 \quad \text { with } \quad \gamma_{k}^{2}=\left(\alpha_{n}^{2}+\iota \frac{u_{k}}{a_{f}} \alpha_{n}+\frac{p}{a_{f}}\right)
$$

The transformed equations (14) and (15) apply for any point in the solid walls or in the fluid sublayer respectively. The interface conditions (6) and the in-plane boundary conditions (3) and (4) are also transformed in the same way. We introduce $\tilde{\bar{\varphi}}$, the Fourier and Laplace transform of the transverse heat flux $\varphi$ (in $y$ direction). So, integration of equations (14) and (15) leads to an analytical solution, in a quadrupolar form [15], of equations (11) to (8) assuming the parameterization of the velocity distribution, which yields :

$$
\left[\begin{array}{l}
\tilde{\bar{\theta}}_{n} \\
\tilde{\bar{q}}_{n}
\end{array}\right]_{h}=\left[\begin{array}{cc}
\mathcal{A}_{n} & \mathcal{B}_{n} \\
\mathcal{C}_{n} & \mathcal{D}_{n}
\end{array}\right]\left[\begin{array}{c}
\tilde{\bar{\theta}}_{n} \\
0
\end{array}\right]_{c}
$$

where

$$
\left[\begin{array}{cc}
\mathcal{A}_{n} & \mathcal{B}_{n} \\
\mathcal{C}_{n} & \mathcal{D}_{n}
\end{array}\right]=\boldsymbol{H}_{1} \boldsymbol{S}_{1 n} \boldsymbol{F}_{n} \boldsymbol{S}_{2 n} \boldsymbol{H}_{2}
$$

and

$$
\boldsymbol{F}_{n}=\prod_{k=1}^{K} \boldsymbol{F}_{k n}
$$


where the indices $h$ and $c$ denote respectively the external faces.

$$
\boldsymbol{H}_{i}=\left[\begin{array}{cc}
1 & 0 \\
h_{i} & 1
\end{array}\right], \quad \boldsymbol{S}_{i n}=\left[\begin{array}{cc}
A_{i n} & B_{i n} \\
C_{i n} & A_{i n}
\end{array}\right] \quad \text { and } \quad \boldsymbol{F}_{k n}=\left[\begin{array}{ll}
A_{k n} & B_{k n} \\
C_{k n} & A_{k n}
\end{array}\right]
$$

$A_{\text {in }}=\cosh \left(\beta_{n} e_{i}\right), \quad B_{\text {in }}=\sinh \left(\beta_{n} e_{i}\right) /\left(\lambda_{s} \beta_{n}\right)$ et $C_{\text {in }}=\left(\lambda_{s} \beta_{n}\right) \sinh \left(\beta_{n} e_{i}\right)$, for $i \equiv s_{1}, s_{2}$.

$A_{k n}=\cosh \left(\gamma_{n} e_{k}\right), B_{k n}=\sinh \left(\gamma_{n} e_{k}\right) /\left(\lambda_{f} \gamma_{n}\right)$ et $C_{k n}=\left(\lambda_{f} \gamma_{n}\right) \sinh \left(\gamma_{n} e_{k}\right)$, for $k=1$ to $K$.

The internal conditions on the $w h$ and $w c$ faces (see Figure 1) as well as on the interfaces between each sublayer $k$ can be written as a function of the external $c$ face :

Internal hot face $(w h)$ :

$$
\left[\begin{array}{c}
\tilde{\bar{\theta}}_{n} \\
\tilde{\bar{\varphi}}_{n}
\end{array}\right]_{w h}=\boldsymbol{F}_{n} \boldsymbol{S}_{2 n} \boldsymbol{H}_{2}\left[\begin{array}{c}
\tilde{\bar{\theta}}_{n} \\
0
\end{array}\right]_{c}=\left[\begin{array}{cc}
\mathcal{A}_{n}^{w h} & \mathcal{B}_{n}^{w h} \\
\mathcal{C}_{n}^{w h} & \mathcal{D}_{n}^{w h}
\end{array}\right]\left[\begin{array}{c}
\tilde{\bar{\theta}}_{n} \\
0
\end{array}\right]_{c}
$$

Internal cold face $(w c)$ :

$$
\left[\begin{array}{c}
\tilde{\bar{\theta}}_{n} \\
\tilde{\bar{\varphi}}_{n}
\end{array}\right]_{w c}=\boldsymbol{S}_{2 n} \boldsymbol{H}_{2}\left[\begin{array}{c}
\tilde{\bar{\theta}}_{n} \\
0
\end{array}\right]_{c}=\left[\begin{array}{cc}
\mathcal{A}_{n}^{w c} & \mathcal{B}_{n}^{w c} \\
\mathcal{C}_{n}^{w c} & \mathcal{D}_{n}^{w c}
\end{array}\right]\left[\begin{array}{c}
\tilde{\bar{\theta}}_{n} \\
0
\end{array}\right]_{c}
$$

Interfaces sublayers $(k)$ :

$$
\left[\begin{array}{c}
\tilde{\bar{\theta}}_{n} \\
\widetilde{\bar{\varphi}}_{n}
\end{array}\right]_{k}=\left(\prod_{k^{\prime}=k+1}^{K} \boldsymbol{F}_{k n}\right) \boldsymbol{S}_{2 n} \boldsymbol{H}_{2}\left[\begin{array}{c}
\tilde{\bar{\theta}}_{n} \\
0
\end{array}\right]_{c}=\left[\begin{array}{cc}
\mathcal{A}_{n}^{k} & \mathcal{B}_{n}^{k} \\
\mathcal{C}_{n}^{k} & \mathcal{D}_{n}^{k}
\end{array}\right]\left[\begin{array}{c}
\tilde{\bar{\theta}}_{n} \\
0
\end{array}\right]_{c}
$$

From the temperature profiles in each sublayer, the bulk temperature profile can be calculated by :

$$
\theta_{b}(x, t) \equiv \frac{1}{2 U_{m} K} \sum_{k=1}^{K} u_{k}\left(\theta\left(x, y_{k}, t\right)+\theta\left(x, y_{k+1}, t\right)\right)
$$




\section{Estimation of internal conditions from measurements on exter- nal faces}

The model (16-19) shows that it is sufficient to know the spectrum of the heat sources $\tilde{\bar{q}}_{n}$ and the structural parameters $\left(e_{1}, e_{2}, e_{f}, U_{m}, \ldots\right)$ in order to be able to retrieve the evolutions of temperatures and heat fluxes everywhere in the system. This formalism corresponds to the resolution of the direct problem.

In the problem of experimental estimation of internal conditions (temperatures and heat fluxes) from the measurements over the external faces, it is difficult to measure the heat source $q(x, t)$. In heat transfer experiments, it is easier to measure temperature. The quadrupole model $(16+19)$ shows that the internal conditions can be derived from the measurement of the temperature field over the hot face, $\theta_{h}$ or over the cold face, $\theta_{c}$. In the mathematical sense, the best choice is to measure the temperature at the place where the signal-to-noise ratio is as large as possible, which is here the temperature over the hot face, $\theta_{h}$.

Since the quadrupole model was written in the Laplace and Fourier domain, therefore the first step of the solution requires estimating the spectrum of experimental $\theta_{h}$.

\subsection{Estimation of the Fourier spectrum of the front face temperature $\theta_{h}$ for each time}

The inverse Fourier transformation over the $[-L ;+L]$ interval (see (13)), is written for the temperature increase of the front "hot" face $\theta_{h}$ at the $x_{i}$ points of the $[-\ell ;+\ell]$ interval, at any time $t_{j}$ :

$$
\theta_{h}\left(x_{i}, t_{j}\right) \approx \frac{1}{2 L} \sum_{n=-n_{h}+1}^{n_{h}} \tilde{\theta}_{n}^{h}\left(t_{j}\right) e^{\ell \alpha_{n} x_{i}}
$$

This can be put under a column-vector/matrix form as $\boldsymbol{\theta}_{\boldsymbol{h}}\left(t_{j}\right)=\boldsymbol{G} \tilde{\boldsymbol{\theta}}_{\boldsymbol{h}}\left(t_{j}\right)$, where $\boldsymbol{\theta}_{\boldsymbol{h}}\left(t_{j}\right)$ is the vector gathering the theoretical noiseless values of the observed $N_{x}$ temperatures at a given time $t_{j}, \tilde{\boldsymbol{\theta}}_{\boldsymbol{h}}\left(t_{j}\right)$ the spectrum vector composed of the $N_{h}$ harmonics used $\left(N_{h} \leq N_{x}\right)$ at the same time, and $\boldsymbol{G}$ the $N_{x} \times N_{h}$ matrix formed with the $e^{\iota \alpha_{n} x_{i}}$ coefficients :

$$
G_{i j}=e^{\iota \alpha_{-n_{h}+j} x_{i}} \quad \text { for } \quad i=1 \text { to } N_{x} \quad \text { and } \quad j=1 \text { to } N_{h}
$$


The Fourier spectrum of the corresponding experimental profile $\boldsymbol{\theta}_{\boldsymbol{h}}^{\boldsymbol{e x p}}\left(t_{j}\right)$ can be estimated through a least squares minimization of :

$$
\begin{gathered}
\hat{\tilde{\boldsymbol{\theta}}}_{h}\left(t_{j}\right)=\arg \left(\min \left(J\left(\tilde{\boldsymbol{\theta}}_{h}\left(t_{j}\right)\right)\right)\right)=\left(\boldsymbol{G}^{*} \boldsymbol{G}\right)^{-1} \boldsymbol{G}^{*} \boldsymbol{\theta}_{\boldsymbol{h}}^{\text {exp }}\left(t_{j}\right) \\
\text { where } \quad J\left(\tilde{\boldsymbol{\theta}}_{\boldsymbol{h}}\left(t_{j}\right)\right)=\left\|\boldsymbol{r}_{k}\left(\tilde{\boldsymbol{\theta}}_{\boldsymbol{h}}\left(t_{j}\right)\right)\right\|^{2} \\
\text { with } \quad \boldsymbol{r}_{k}\left(\tilde{\boldsymbol{\theta}}_{\boldsymbol{h}}\left(t_{j}\right)\right)=\boldsymbol{\theta}_{\boldsymbol{h}}^{\boldsymbol{e x p}}\left(t_{j}\right)-\boldsymbol{G} \tilde{\boldsymbol{\theta}}_{\boldsymbol{h}}\left(t_{j}\right)
\end{gathered}
$$

where $\hat{\tilde{\boldsymbol{\theta}}}_{h}$ is the ordinary least square estimate of $\tilde{\boldsymbol{\theta}}_{h}, J$ the ordinary least squares sum, $\boldsymbol{r}_{k}$ the residual vector at time $t_{j}, \boldsymbol{G}^{*}$ the conjugate transpose of complex matrix $\boldsymbol{G}$. This can also be written using the Singular Matrix Decomposition (SVD) of matrix $\boldsymbol{G}$, which is square if $N_{h}=N_{x}$ :

$$
\hat{\tilde{\boldsymbol{\theta}}}_{h}\left(t_{j}\right)=\boldsymbol{V} \boldsymbol{\Sigma}^{-1} \boldsymbol{U}^{*} \boldsymbol{\theta}_{\boldsymbol{h}}^{\boldsymbol{e x p}}\left(t_{j}\right) \quad \text { where } \quad \boldsymbol{G}=\boldsymbol{U} \boldsymbol{\Sigma} \boldsymbol{V}^{*}
$$

where

$$
\begin{gathered}
\boldsymbol{\Sigma}=\operatorname{diag}\left(\Sigma_{1}, \quad \Sigma_{2}, \quad \ldots, \quad \Sigma_{N_{x}}\right) \\
\Sigma_{1} \geqslant \Sigma_{2} \geqslant \ldots \geqslant \Sigma_{N_{x}-1} \geqslant \Sigma_{N_{x}}>0 \\
\boldsymbol{U}^{*} \boldsymbol{U}=\boldsymbol{U} \boldsymbol{U}^{*}=\boldsymbol{V} \boldsymbol{V}^{*}=\boldsymbol{V}^{*} \boldsymbol{V}=\boldsymbol{I}_{N_{x}} \\
\boldsymbol{U}=\left[\begin{array}{lllll}
\boldsymbol{U}_{1}, \quad \boldsymbol{U}_{2}, \quad \ldots, \quad \boldsymbol{U}_{N_{x}}
\end{array}\right] \quad \text { and } \quad \boldsymbol{V}=\left[\begin{array}{llll}
\boldsymbol{V}_{1}, & \boldsymbol{V}_{2}, \quad \ldots, & \boldsymbol{V}_{N_{x}}
\end{array}\right]
\end{gathered}
$$

Here $\boldsymbol{\Sigma}$ is the diagonal matrix composed of the singular values $\Sigma_{i}$ of $\boldsymbol{G}$ (for $i=1$ to $N_{x}$ ), $\boldsymbol{U}$ the matrix of its $N_{x}$ left singular (column) vectors $\boldsymbol{U}_{\boldsymbol{i}}$ and $\boldsymbol{V}$ the matrix of its $N_{x}$ right singular (column) vectors $\boldsymbol{V}_{\boldsymbol{i}}$.

In the cas where the matrix to be inverted $\boldsymbol{G}$ or $\boldsymbol{\Sigma}$ is ill-conditioned $\left(\Sigma_{1} / \Sigma_{N_{x}}>>1\right)$, the experimental spectrum estimation using model $(22)$ or (23) becomes unstable [3]. To overcome this difficulty, that is to make this estimate stable, one has to regularize. In other words, one has to modify the matrix to be inverted to make it well conditioned [16]. Several regularization techniques exist. In this work only the Truncated Singular Value Decomposition $(T S V D)$ technique [17, 18, 19, 20] is used. The TSVD as implied by its name, consists in truncating the smallest singular values that are responsible for high values of the condition number of $\boldsymbol{G}$ or $\boldsymbol{\Sigma}$. The truncated version of this estimator is :

$$
\begin{gathered}
\hat{\tilde{\boldsymbol{\theta}}}_{\boldsymbol{h}, \alpha_{x}}\left(t_{j}\right)=\boldsymbol{V} \boldsymbol{\Sigma}_{\alpha_{x}}^{-1} \boldsymbol{U}^{*} \boldsymbol{\theta}_{\boldsymbol{h}}^{\boldsymbol{e x p}}\left(t_{j}\right) \\
\text { with } \Sigma_{\alpha_{x}}^{-1}=\operatorname{diag}\left(\Sigma_{1}^{-1}, \Sigma_{2}^{-1}, \ldots, \Sigma_{\alpha_{x}}^{-1}, 0, \ldots, 0\right)
\end{gathered}
$$


where $\alpha_{x}$ is the regularization parameter whose integer value can vary from 1 to $N_{h}$. Between the two limits, there is an optimum which can be found using Morozov's discrepancy principle [16] or by the L-curve [21]. Here Morozov's discrepancy principle is used. It consists to get a Root Mean Square Residual (RMSR) that cannot be lower than the measurement noise (level of residuals slightly higher than the measurement noise) :

$$
\operatorname{RMSR}\left(\alpha_{x}\right) \geq \sigma \quad \text { with } \quad R M S R\left(\alpha_{x}\right)=\sqrt{\frac{\boldsymbol{r}_{k}\left(\tilde{\boldsymbol{\theta}}_{\boldsymbol{h}, \alpha_{x}}\left(t_{j}\right)\right)^{T} \boldsymbol{r}_{k}\left(\tilde{\boldsymbol{\theta}}_{\boldsymbol{h}, \alpha_{x}}\left(t_{j}\right)\right)}{N_{x}}}
$$

where $\sigma$ is the noise standard deviation.

\subsection{Time inversion using regularized deconvolution of the Fourier harmonics of the front face temperature}

Once the Fourier spectrum vector $\hat{\tilde{\boldsymbol{\theta}}}_{h, \alpha_{x}}\left(t_{j}\right)$, of size $N_{h} \times 1$, of the front face temperature $\boldsymbol{\theta}_{h}\left(t_{j}\right)$, has been estimated for each time $t_{j}=j \Delta t$ (for $j=1$ to $N_{t}$ ), all the different harmonics can be recast into $N_{h}$ column-vectors $\hat{\tilde{\boldsymbol{\theta}}}_{n}^{h}$, of size $N_{t} \times 1$, whose components are equals to the TSVD estimation of $\hat{\tilde{\theta}}_{n}^{h}\left(t_{j}\right)$, as :

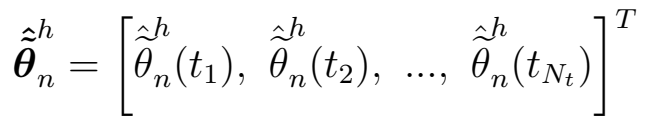

Since the system of equations (1) to (8) is linear with time invariant coefficients, the simple product in the Laplace domain is a convolution product in the time domain. Using equations 16 to 19, the unknown spectrum of temperature or heat flux at a given face, let's call it $\tilde{y}_{n}$ here, can be calculated from the Fourier transform of front face temperature $\tilde{\theta}_{n}^{h}$ as :

$$
\begin{aligned}
\tilde{\bar{y}}_{n} & =\tilde{\overline{\mathcal{H}}}_{n} \tilde{\bar{\theta}}_{n}^{h} \\
\Longrightarrow \tilde{y}_{n}(t) & =\tilde{\mathcal{H}}_{n}(t) * \tilde{\theta}_{n}^{h}(t) \\
& =\int_{0}^{t} \tilde{\mathcal{H}}_{n}\left(t-t^{\prime}\right) \tilde{\theta}_{n}^{h}\left(t^{\prime}\right) \mathrm{d} t^{\prime}
\end{aligned}
$$

where the star symbol $(*)$ designates the convolution product in the time domain and $\tilde{\mathcal{H}}_{n}$ is the Fourier transform of the impulse response, that is the original of transfer function $\overline{\mathcal{H}}(p)$. The latter can be called a transimttance, $\mathcal{H} \equiv W$ if the response corresponds to a temperature $y \equiv \theta$ and it is called 
an admittance, $\mathcal{H} \equiv Y$ if the response corresponds to a heat flux $y \equiv \varphi[1][2]$.

The convolution integral in equation (27c), is calculated through a numerical quadrature. This can be put under a column-vector/matrix form :

$$
\hat{\tilde{\boldsymbol{y}}}_{n}=\boldsymbol{M}\left(\tilde{\mathcal{H}}_{n}\right) \hat{\tilde{\boldsymbol{\theta}}}_{n}^{h}
$$

where $\boldsymbol{M}$ (.) is a (square) matrix function of a column vector, here a lower triangular Toeplitz matrix based on the parameterization of a function on a basis of piecewise constant functions. It is defined as :

$$
\boldsymbol{M}(\boldsymbol{\psi})=\Delta t\left[\begin{array}{ccccc}
\psi_{1} & 0 & 0 & \cdots & 0 \\
\psi_{2} & \psi_{1} & 0 & \ddots & \vdots \\
\psi_{3} & \psi_{2} & \psi_{1} & \ddots & \vdots \\
\vdots & \vdots & \vdots & \ddots & \vdots \\
\psi_{N_{t}} & \psi_{N_{t}-1} & \psi_{N_{t}-2} & \cdots & \psi_{1}
\end{array}\right] \quad \text { with } \quad \boldsymbol{\psi}=\left[\begin{array}{c}
\psi_{1} \\
\psi_{2} \\
\psi_{3} \\
\vdots \\
\psi_{N_{t}}
\end{array}\right]
$$

where $\psi_{j}=\frac{1}{\Delta t} \int_{t_{j-1}}^{t_{j}} \psi(t) d t \simeq \frac{1}{2}\left(\psi\left(t_{j-1}\right)+\psi\left(t_{j}\right)\right)$ and $t_{j}=j \Delta t$ for $j=1$ to $N_{t}$ and $\psi\left(t_{0}=0\right)=0$. The temperture or heat flux profiles over a given face et at each time $t_{j}$, can be calculated from its Fourier spectrum vector, see equations $21 \mathrm{a}$ and $21 \mathrm{~b}$ :

$$
\hat{\boldsymbol{y}}\left(t_{j}\right)=\boldsymbol{G} \hat{\tilde{\boldsymbol{y}}}\left(t_{j}\right)
$$

Let us note that the calculation of each harmonics of the internal and rear face temperature or heat flux $\left(y \equiv \theta_{w h}, \theta_{w c}, \theta_{k}, \theta_{c}, \varphi_{w h}\right.$ or $\left.\varphi_{w c}\right)$ at any time, see (28), and hence its corresponding space profile, see (30), corresponds to the solution of a direct and well-posed problem (convolution problem). It is not the case for the heat source profile, $\boldsymbol{q}$, estimation which corresponds to a deconvolution problem, see 16 :

$$
\begin{gathered}
\hat{\tilde{\boldsymbol{q}}}_{n}=\left[\mathcal{\mathcal { K }}_{N_{t}}\right]^{-1} \boldsymbol{M}\left(\tilde{\mathcal{H}}^{2}{ }_{n}\right) \hat{\tilde{\boldsymbol{\theta}}}_{n}^{h} \text { where } \quad \mathcal{K}_{N_{t}}=\boldsymbol{M}\left(\tilde{\mathcal{H}}^{1}{ }_{n}\right) \\
\text { with } \quad \tilde{\mathcal{H}}_{n}^{1}(t) \equiv L^{-1}\left(\frac{1}{\mathcal{C}_{n}}\right) \text { and } \quad \tilde{\mathcal{H}}_{n}^{2}(t) \equiv L^{-1}\left(\frac{1}{\mathcal{A}_{n}}\right)
\end{gathered}
$$

where $L^{-1}$ is the inverse Laplace transform, which can be calculated numerically by Stehfest's algorithm [22, 23]. Since the measured temperature $\theta_{h}$ 
is noisy, the heat source estimation, see (31) is an ill-posed problem. In this case, a time regularization by $T S V D$ is possible :

$$
\hat{\tilde{\boldsymbol{q}}}_{n}=\mathcal{K}_{\alpha_{t}}^{\dagger} \boldsymbol{M}\left(\tilde{\mathcal{H}}^{\mathbf{2}}{ }_{n}\right) \hat{\tilde{\boldsymbol{\theta}}}_{n}^{h}
$$

with

$$
\mathcal{K}_{\alpha_{t}}^{\dagger}=\boldsymbol{V} \boldsymbol{\Sigma}_{\alpha_{t}}^{-1} \boldsymbol{U}^{*} \quad \text { where } \quad \mathcal{K}_{N_{t}}=\boldsymbol{M}\left(\tilde{\mathcal{H}}^{1}{ }_{n}\right)=\boldsymbol{U} \boldsymbol{\Sigma}_{N_{t}} \boldsymbol{V}^{*}
$$

where $\mathcal{K}_{\alpha_{t}}^{\dagger}$ is the pseudo-inverse of $\mathcal{K}_{N_{t}}$, obtained by its TSVD form above, keeping $\alpha_{t}$ singular values. $\alpha_{t}$ is hyper-parameter corresponding to the temporal $T S V D$ regularization. The temperatures, heat flux and corresponding heat source, can be estimated from the information on the external face $\theta_{h}$ as listed in (Table 1. 


\section{Results of the validation of the estimation technique on synthe- tic profiles}

The system presented above, see section 2.1 was simulated here by COMSOL [24]. The temperature responses on the front, rear and internes faces $\left(\theta_{h}, \theta_{c}, \theta_{w h}\right.$ and $\theta_{w c}$ respectively) correponding to surface heat source $q(x, t)$ were calculated at each time $t_{j}$ where $j=1$ to $N_{t}$ with $N_{t}=t_{f} / \triangle t$, where $N_{t}$ is observation number and $\triangle t$ is the time step .In this simulation, we take $\Delta t=1 \mathrm{~s}$ and $N_{t}=500$. The simulation parameters are given in Table 2 and Table 3 . Grid independence tests have been performed using several grid densities. In the present study triangular elements were used with 2152 degrees of freedom. The surface heat source $q(x, t)$ has been assumed separable and uniform in space between $x_{1}$ and $x_{2}$ here. So it can be written as :

$$
q(x, t)=Q(t)\left[\mathbb{H}\left(x-x_{1}\right)-\mathbb{H}\left(x-x_{2}\right)\right] \frac{1}{S}
$$

where $Q(t)$ is the time distribution of $q(x, t)$ over a surface $S$, here a rectangular surface of length $x_{2}-x_{1}$. Here $Q(t)$ is a step function with $Q(t<0)=0$ and $Q(t \geq 0)=Q^{s s}$ where $s s$ designates the steady state. $\mathbb{H}$ designates the Heaviside function. The initial temperature $T(x, y, t \leq 0)$ of the system is assumed to be uniform and equal to the ambient temperature $T_{\infty}=20^{\circ} \mathrm{C}$. The lateral heat loss coefficient is taken here uniform $\left(h_{1}=h_{2}=10 \mathrm{~W} \cdot \mathrm{m}^{-2} \cdot \mathrm{K}^{-1}\right)$. The steady state surface heat source is set to $q^{s s}(x)=400 \mathrm{~W} \cdot \mathrm{m}^{-2}$. The corresponding average velocity $U_{m}$, Reynolds number $R e$ and the Péclet number $P e$ are given in Table 4. Let us note that the Péclet number, $P e$, is equal to 1.4, which means that neither diffusion nor axial advection can be neglected in the heat equation for the fluid.

TABLE 2: Standard thermophysical parameters.

\begin{tabular}{ccccc}
\hline $\begin{array}{c}\lambda_{s} \\
\left(W \cdot m^{-1} \cdot K^{-1}\right)\end{array}$ & $\begin{array}{c}\lambda_{f} \\
\left(W \cdot m^{-1} \cdot K^{-1}\right)\end{array}$ & $\begin{array}{c}\rho c_{s} \\
\left(k J \cdot m^{-3} \cdot K^{-1}\right)\end{array}$ & $\begin{array}{c}\rho c_{f} \\
\left(k J \cdot m^{-3} \cdot K^{-1}\right)\end{array}$ & $\begin{array}{c}\nu_{f} \\
\left(m^{2} \cdot s^{-1}\right)\end{array}$ \\
\hline 0.215 & 0.63 & 1440 & 4186 & $1.10^{-6}$ \\
\hline
\end{tabular}

The exact front face temperature profile at each time $t_{j}$ obtained by COMSOL $\boldsymbol{\theta}_{h}^{\text {exact }}\left(t_{j}\right)$ is considered here as an experimental profile (noisy profile) after adding an independent identically distributed noise $\varepsilon$ characterized 
TABLE 3: Standard geometrical parameters.

\begin{tabular}{ccccccc}
\hline $2 \ell$ & $K_{v}$ & $x_{1}$ & $x_{2}$ & $e_{1}=e_{2}=2 e_{f}$ & $K$ & $N_{x}=N_{h}$ \\
$(m m)$ & - & $(m m)$ & $(m m)$ & $(m m)$ & - & - \\
\hline 65 & 2 & -6 & 6 & 2 & 5 & 600 \\
\hline
\end{tabular}

TABLE 4: The average velocity and corresponding dimensionless numbers.

\begin{tabular}{ccc}
\hline$U_{m}\left(m \cdot s^{-1}\right)$ & $R e=2 U_{m} e_{f} / \nu_{f}$ & $P e=2 U_{m} e_{f} / a_{f}$ \\
\hline $10^{-4}$ & 0.2 & 1.4 \\
\hline
\end{tabular}

by a standard deviation $\left(\sigma_{\text {noise }}=0.03^{\circ} \mathrm{C}\right)$ for $N_{x}$ equidistant points on the interval $[-\ell ;+\ell]$ of the front face :

$$
\boldsymbol{\theta}_{h}^{\text {exp }}\left(t_{j}\right)=\boldsymbol{\theta}_{h}^{\text {exact }}\left(t_{j}\right)+\boldsymbol{\varepsilon}
$$

Using this noised "experimental" profile $\boldsymbol{\theta}_{h}^{e x p}\left(t_{j}\right)$ and the inversion technique presented in section (3), the internal, bulk and rear face temperature profiles are estimated. The experimental (from the numerical experiment) and estimated temperature profiles are presented successively. The experimental and recalculated front face temperature, $T_{h}$ is shown in Figure 3 , the exact and estimated internal hot face temperature, $T_{w h}$ in Figure 4 , the exact and estimated bulk temperature, $T_{b}$ in Figure 5, the exact and estimated internal cold face temperature, $T_{w c}$ in Figure 6 and finally the exact and estimated rear (cold) face temperature, $T_{c}$ in Figure 7. The profiles are represented at times $t=80,240$, and $400 \mathrm{~s}$. It is clear that there is a very good correlation between the exact temperature profiles obtained by COMSOL (direct problem) and their estimated counterparts obtained in a semi-analytical way using adhoc transfer functions (inverse problem).

The heat source as well as hot face flux are also estimated in the same way. The heat source and of its exact distribution, $q$ is shown in Figure 8 and the estimated external hot face flux $\varphi_{h}$ is shown in Figure 9, Figure 8 shows that the heat source can be estimated fairly accurately (spatial door function with intensity of $400 \mathrm{~W} \cdot \mathrm{m}^{-2}$ ) whatever the considered time. The spatial and temporal regularization hyperparameters of each estimation $\left(\alpha_{x}\right.$ for recalculated $T_{h}$ and $\alpha_{t}$ for $q_{h}$ ) are indicated in the legend of the corres- 
ponding figures. The obtained results are encouraging, allowing us to apply this method of inversion to real measurements, as can be seen in the next section.

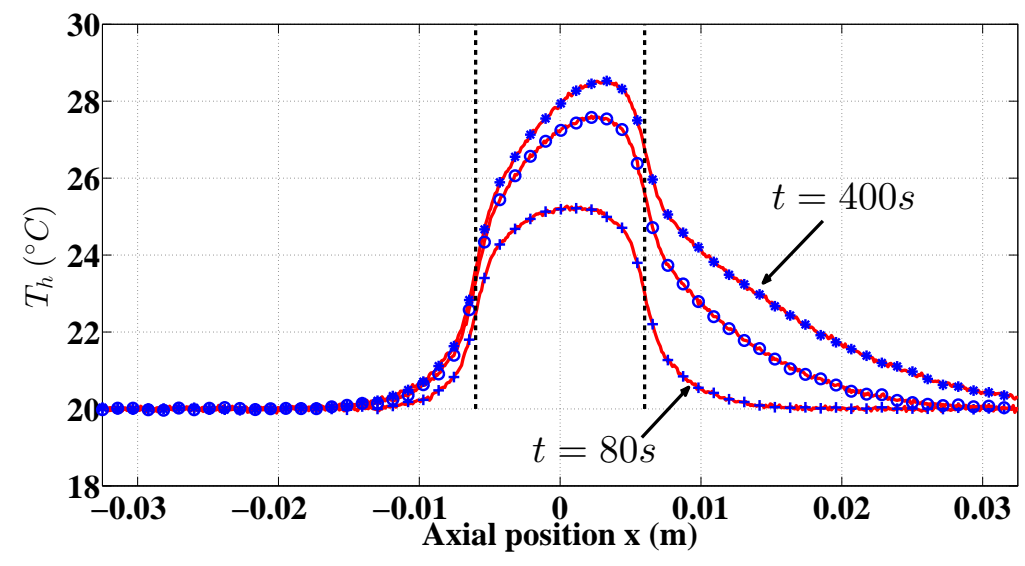

Figure 3: Noised "experimental" (full lines) and recalculated (symbols and dots) front face temperature $T_{h}(x, t)$ at times $t=80,240$ and $400 s\left(\alpha_{x}=310\right.$ for $N_{x}=600$ ).

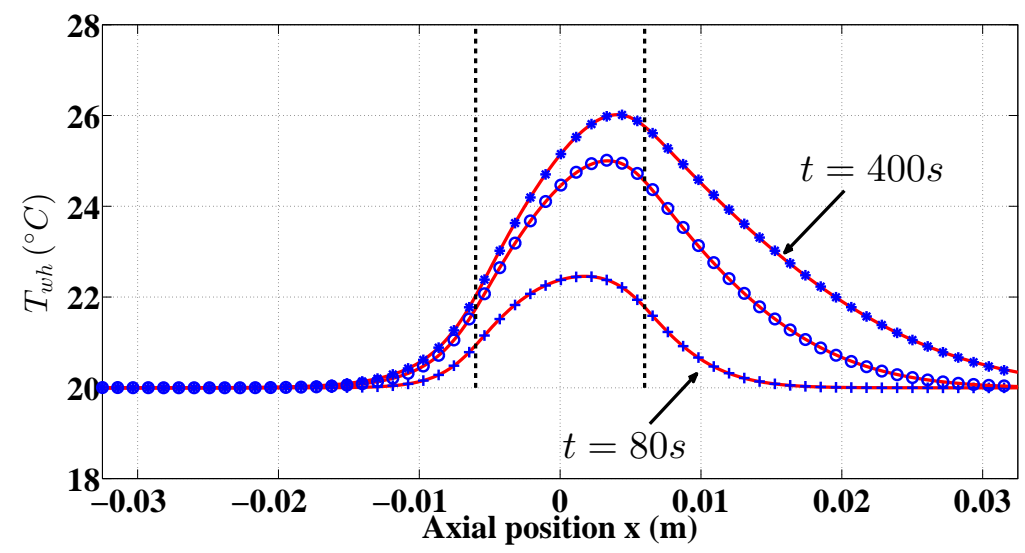

FIGURE 4: Exact (full lines) and estimated (symbols and dots) internal hot face temperature $T_{w h}(x, t)$ at times $t=80,240$ and $400 s$. 


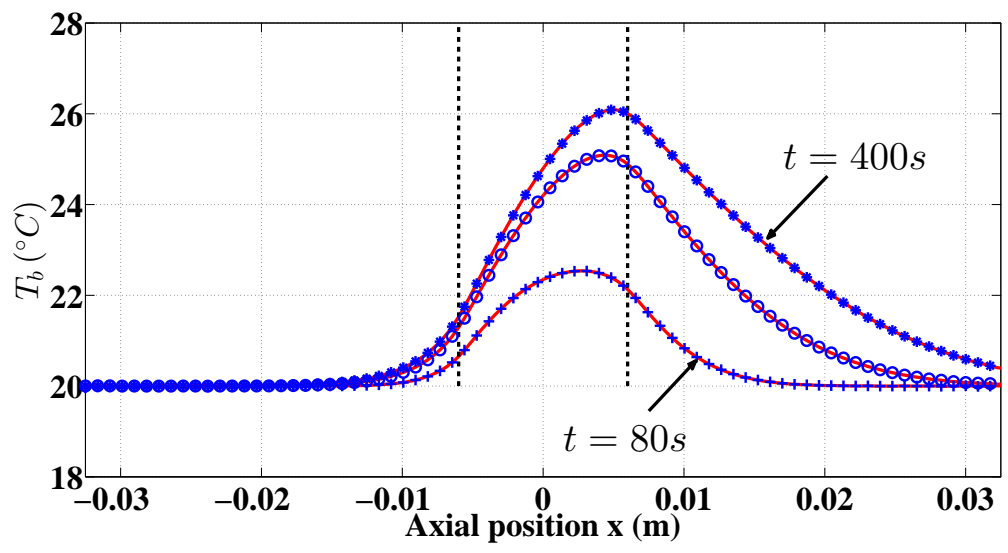

FIGURE 5: Exact (full lines) and estimated (symbols and dots) bulk temperature $T_{b}(x, t)$ at times $t=80,240$ and $400 s$.

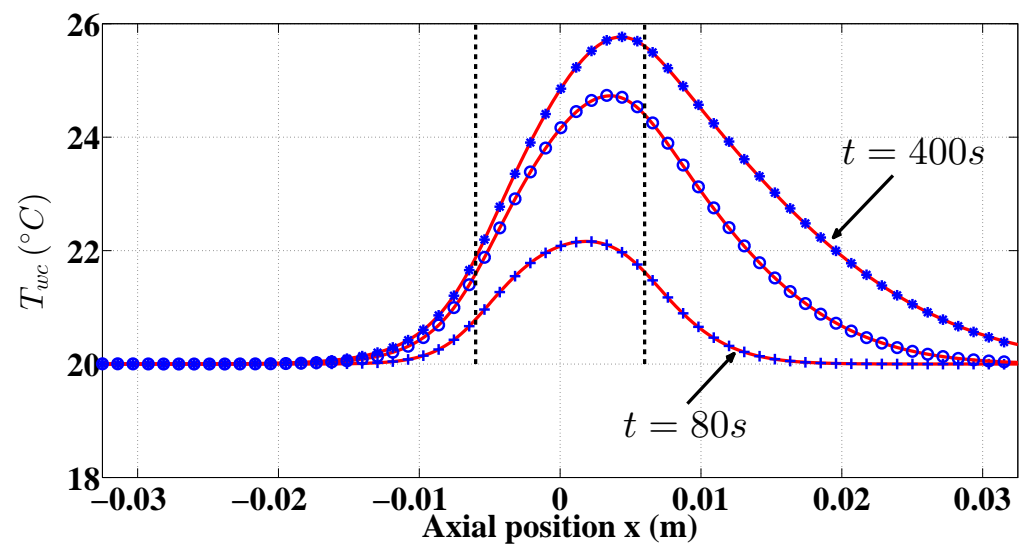

Figure 6: Exact (full lines) and estimated (symbols and dots) internal cold face temperature $T_{w c}(x, t)$ at times $t=80,240$ and $400 s$.

\section{Results of experimental estimation}

\subsection{Experimental setup}

A sketch of the cross section of the channel is presented in Figure 10. The bench is composed of two polycarbonate (conductivity $\lambda_{s}$, volumetric heat $\rho c_{s}$ ) plates that are the outer walls of a three-layer system (thicknesses $e_{1}$ 


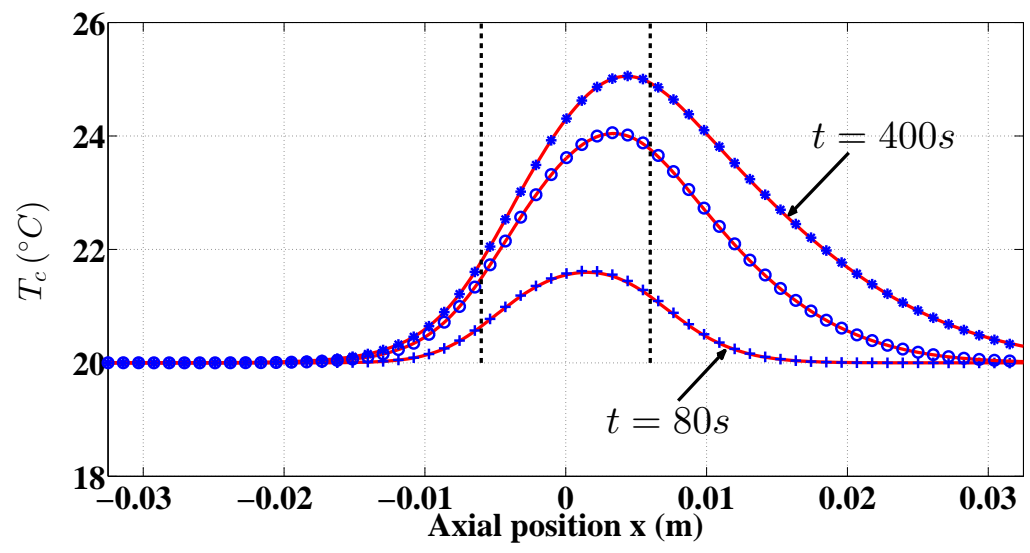

FIGURE 7: Exact (full lines) and estimated (symbols and dots) rear (cold) face temperature $T_{c}(x, t)$ at times $t=80,240$ and $400 s$.

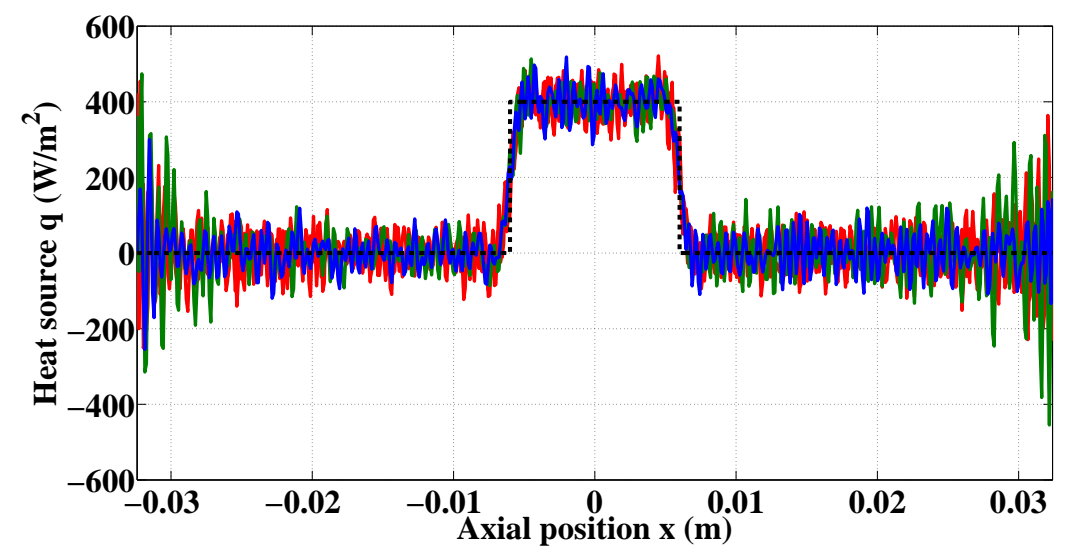

Figure 8: Exact (dotted lines) and estimated (full lines) front face heat source $q(x, t)$ at times $t=80 \mathrm{~s}$ (red), 240s (bleu) and 400s (green) ( $\alpha_{t}=490$ for $\left.N_{t}=500\right)$.

and $e_{2}$ for the front and rear walls). The constant cross section of the channel is equal to $w e_{f}$, where $e_{f}$ is its thickness and $w$ its width in the dimension normal to this figure. Two plenum chambers of larger thicknesses are located upstream and downstream the constant section channel. The total length of the channel, which corresponds to the distance between these two chambers 


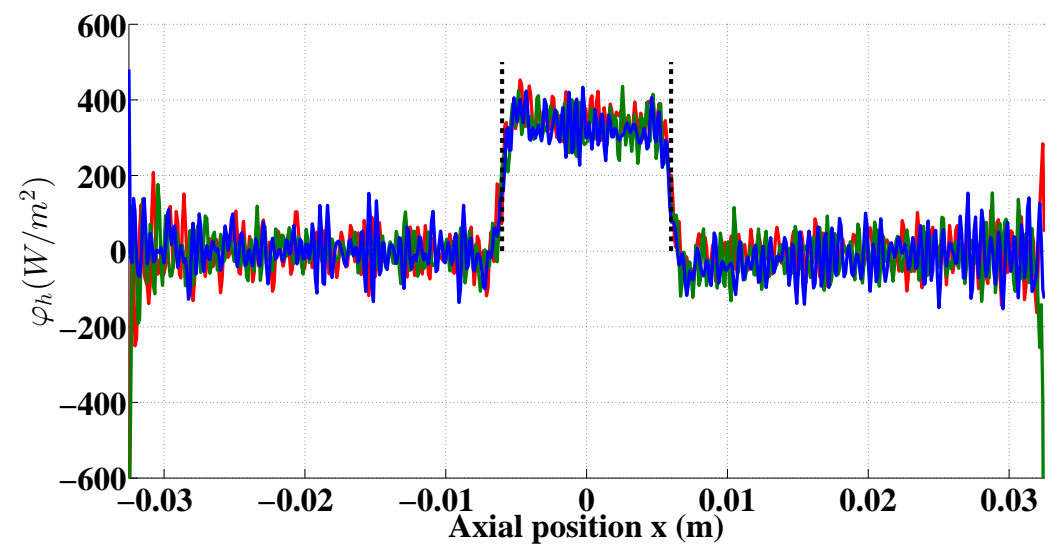

Figure 9: Estimated front face flux distributions $\varphi_{h}(x, t)$ at times $t=80 \mathrm{~s}$ (red), 240s (bleu) and 400s (green).

is $2 \ell_{c}$, while its length with the above defined constant thicknesses of its two walls is $2 \ell$.

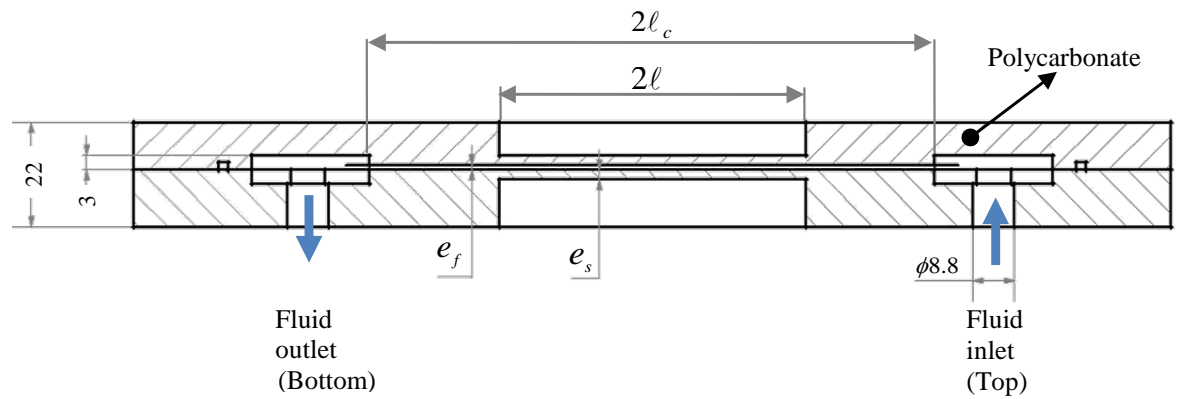

FIGURE 10: Sketch of polycarbonate channel.

The liquid (de-ionized and deaerated water, conductivity $\lambda_{f}$, volumetric heat $\rho c_{f}$ ) is fed through the setup by a serynge pump with a constant average velocity $U_{m}$ and the temperature $T_{i n}$ of the water in the upstream plenum (tranquilization chamber) is adjusted thanks to the set point temperature of the thermostat that is adjusted in order to get a water inlet temperature $T_{\text {in }}=T_{\infty}$, where $T_{\infty}$ is the temperature of the outside environment (air and 
walls of the lab), see Figure 11.

The heating system, whose electrical power (tension $V$ and current $I$ ) is recorded, is detailed in Figure 12a (left) : it is composed of a rectangular foil heating resistance, of area equal to $w \ell_{h}$, which is separated from the front face of the channel by a copper bar, of length $w$ and cross section $e_{h} \ell_{h}$, with a thermocouple set at its center. In order to have a low contact resistance between the copper bar and the channel front face, a $25 \mu \mathrm{m}$ thick of indium foil is set in between. Prior to heating, the syringe pump is activated during a time long enough in order to get the front face temperature monitored by the infrared camera, uniform and equal to $T_{\infty}$. So the incoming liquid temperature, the ambient temperature and the temperature field in the whole system are initially equal, which means that the temperature rise wherever in the system will be caused by Joule effect only. Pictures of the channel as well as of the whole setup are shown in Figure 13. The different geometrical and thermopysical parameters of the system are given in Table 5 and Table 6 respectively.

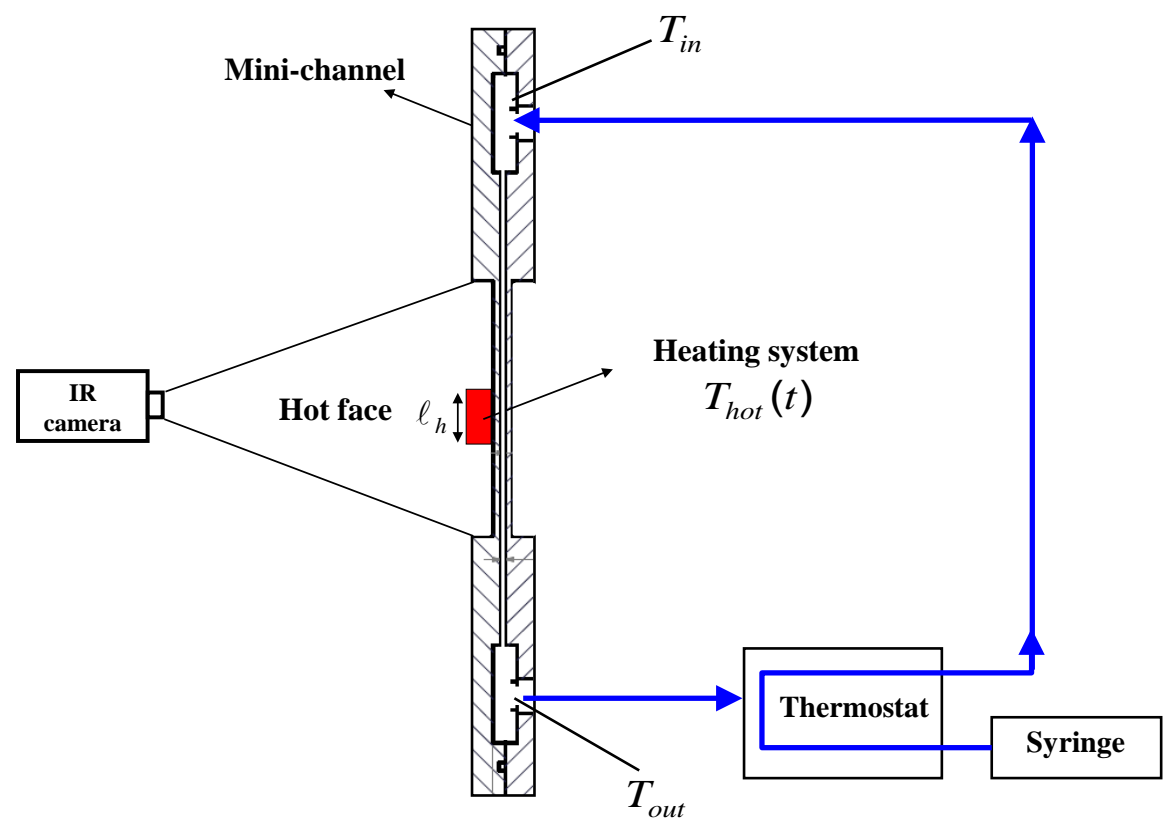

FIGURE 11: Mini channel with hydraulic circuit, infrared and thermocouple. 


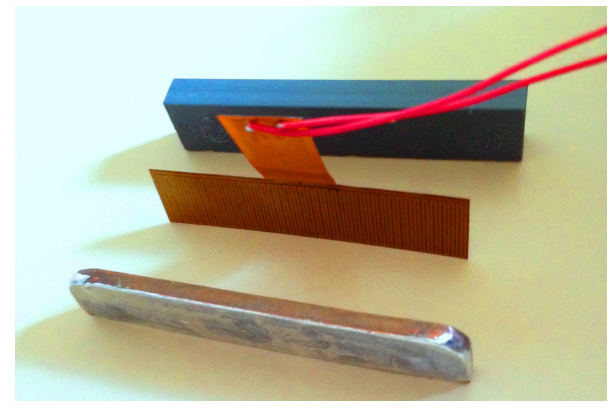

(a) Real

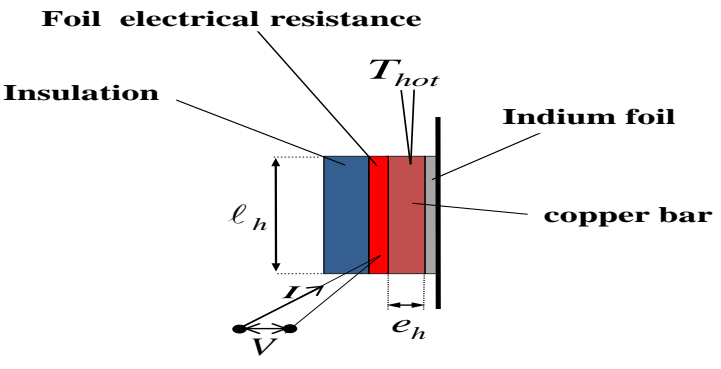

(b) Schema

FIGURE 12: Detail of heating system.

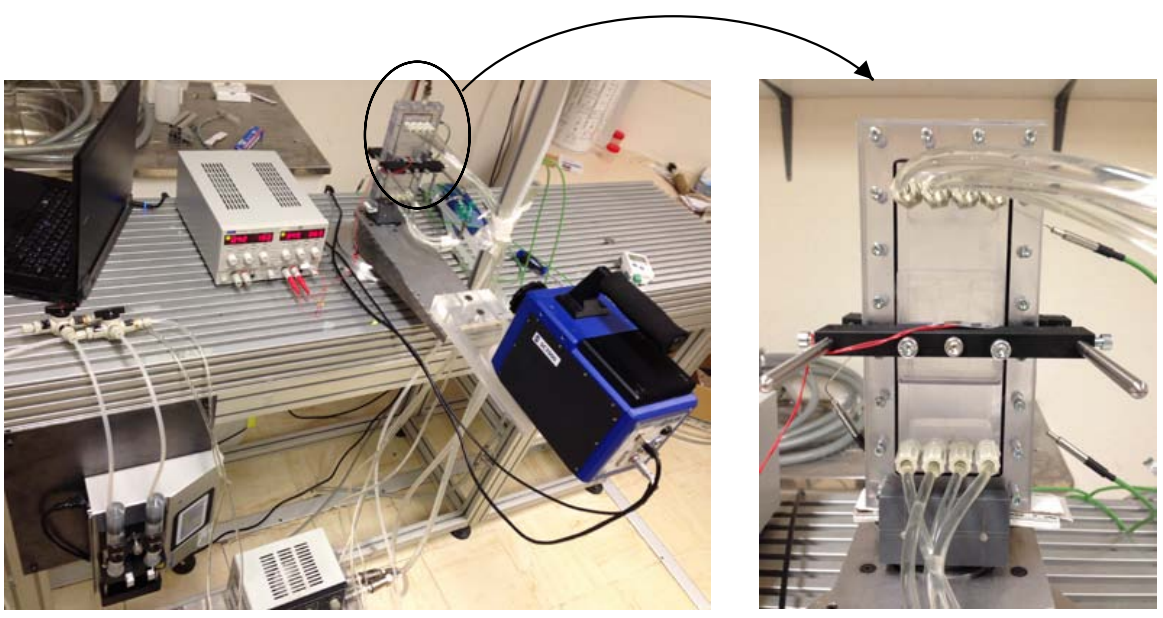

FiguRE 13: Mini channel with hydraulic circuit, and surface heat source..

TABLE 5: Geometrical parameters.

\begin{tabular}{cccccc}
\hline$e_{1}=e_{2}(\mathrm{~mm})$ & $e_{f}(\mathrm{~mm})$ & $2 \ell(\mathrm{mm})$ & $2 \ell_{c}(\mathrm{~mm})$ & $w(\mathrm{~mm})$ & $\ell_{h}(\mathrm{~mm})$ \\
\hline 2 & 1 & 65 & 120 & 50 & 10
\end{tabular}


TABLE 6: Thermopysical parameters.

\begin{tabular}{cccc}
\hline$\lambda_{s}$ & $\rho c_{s}$ & $\lambda_{f}$ & $\rho c_{f}$ \\
$\left(W \cdot m^{-1} \cdot K^{-1}\right)$ & $\left(k J \cdot m^{-3} \cdot K^{-1}\right)$ & $\left(W \cdot m^{-1} \cdot K^{-1}\right)$ & $\left(k J \cdot m^{-3} \cdot K^{-1}\right)$ \\
\hline 0.215 & 1440 & 0.63 & 4186 \\
\hline
\end{tabular}

\subsection{Front face temperature measurement and preprocessing}

For an imposed average water velocity $U_{m}=10^{-4} \mathrm{~m} \cdot \mathrm{s}^{-1}(P e=1.4)$, the temperature field over the hot (front) face, $\theta_{h}(x, t)$ is measured either by the thermocouple located inside the copper bar over the heating length $\ell_{h}$, or by an infrared camera focused on the external (front) surface of the channel right after the initial time when electrical heating starts until $t_{f}=2400 \mathrm{~s}$. A time average of 5 frames, which are acquired with a period of $0.2 s$, followed by a space averaging of the thermographic signal over 5 columns of pixels in the $x$ direction is made, which result in the measurement of a $\mathrm{x}$-temperature profile with a period $\Delta t^{\prime}=1 \mathrm{~s}$, which is the same as the acquisition time step of the thermocouple located inside the copper bar, see Figure 11. The infrared plane array InSb camera FLIR-Cedip Jade II works in the 3.5-5.6 $\mathrm{mm}$ spectral interval. The temperature of the surrounding radiative environment is supposed to be equal to $T_{\infty}$. The uniform temperature of the copper bar is distributed over ficticious "pixels" of the same space step $\triangle x$ as the pixels measured by the infrared camera. Because of the thickness of the heating assembly, see Figure 11 and Figure 13, and of the space transfer function of the camera which acts as a low pass filter (about 5 pixels are necessary to grasp a sharp temperature transition), the pixels in the vicinity of the copper bar cannot be measured and are replaced by a linear interpolation between the copper temperature and the recorded temperature beyond this 5 pixels interval.

\subsection{Experimental results}

We present in Figure 14 the measured and recalculated hot front face temperature only at times $80,400,800,1200$ and 1600 seconds past the start of the electrical step excitation. The chosen time step is $\triangle t=4 \Delta t^{\prime}=4 \mathrm{~s}$ and TSVD regularization has been implemented for the $N_{x}=250$ pixels of the front face temperature over the $]-\ell ;+\ell]$ interval and for $L=2 \ell$ in order to estimate the $N_{h}=N_{x}$ harmonics of the front face temperature spectrum with only $\alpha_{x}=130$ singular values. The estimated hot interface temperature $T_{w h}$, 
the estimated bulk temperature $T_{b}$, the estimated cold interface temperature $T_{w c}$ as well as the estimated rear face temperature $T_{c}$ are plotted respectively in Figure 15 to Figure 17.

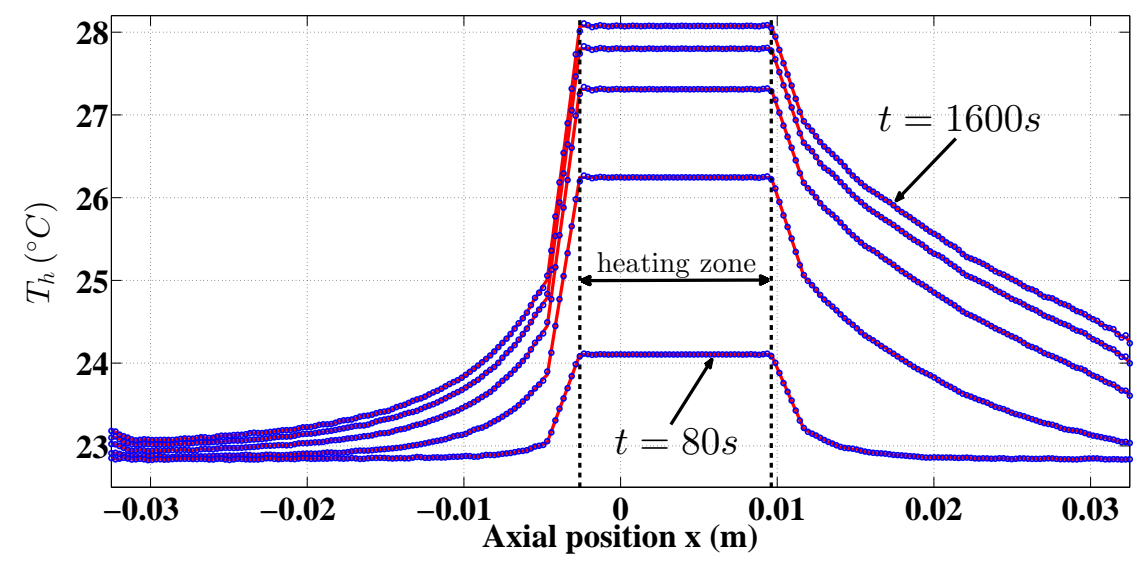

FiguRE 14: Hot front face temperature $T_{h}$ profiles (measured "full lines" and recalculated "circle" with $\alpha_{x}=130$ for $N_{x}=250$ ).

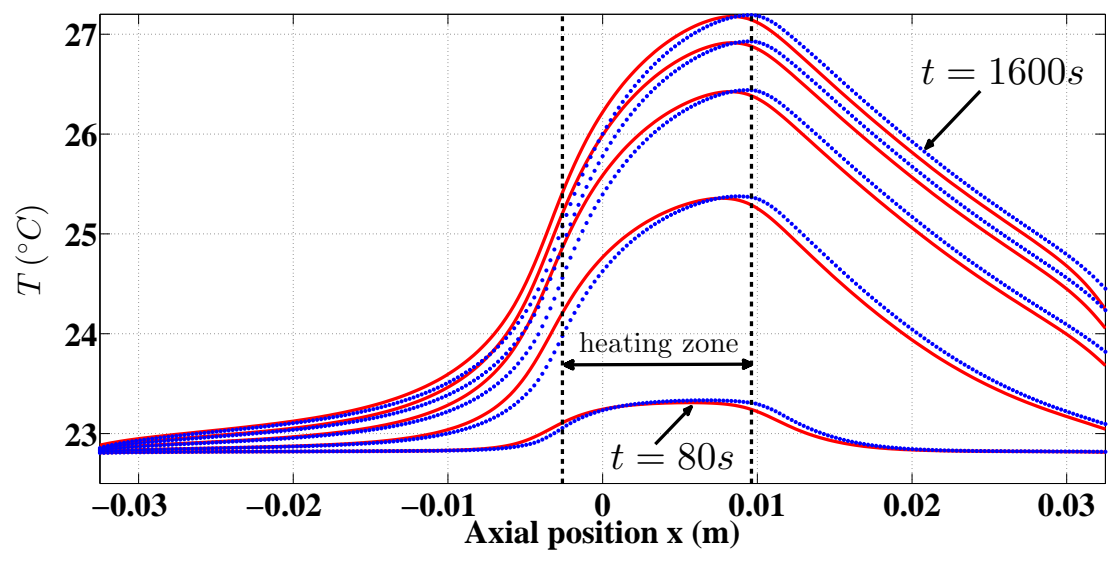

Figure 15: Estimated hot interface temperature $T_{w h}$ "full lines" and bulk fluid temperature $T_{b}$ "circle" profiles. 


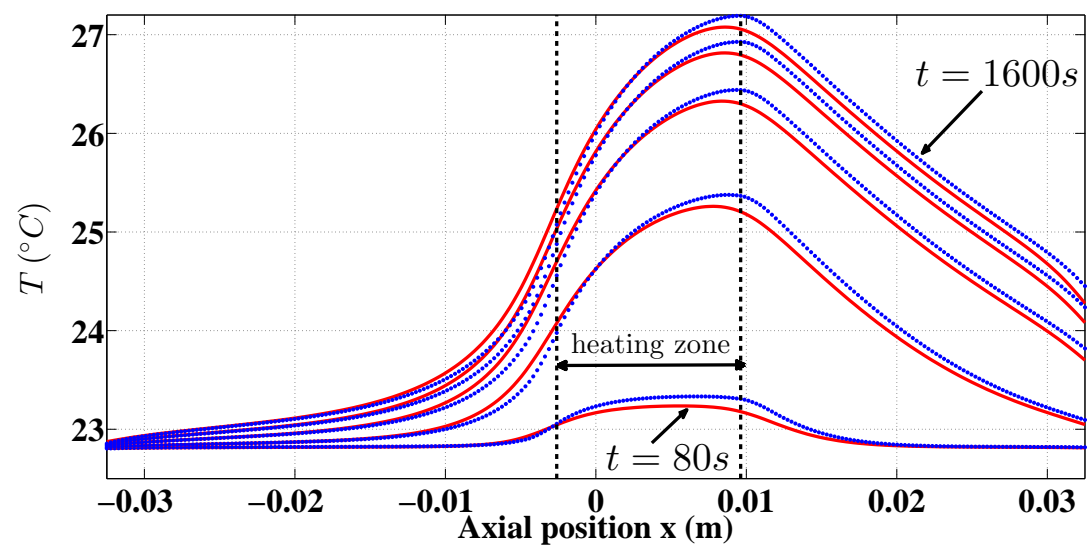

FiguRE 16: Estimated cold interface temperature $T_{w c}$ "full lines" and bulk fluid temperature $T_{b}$ "circle" profiles.

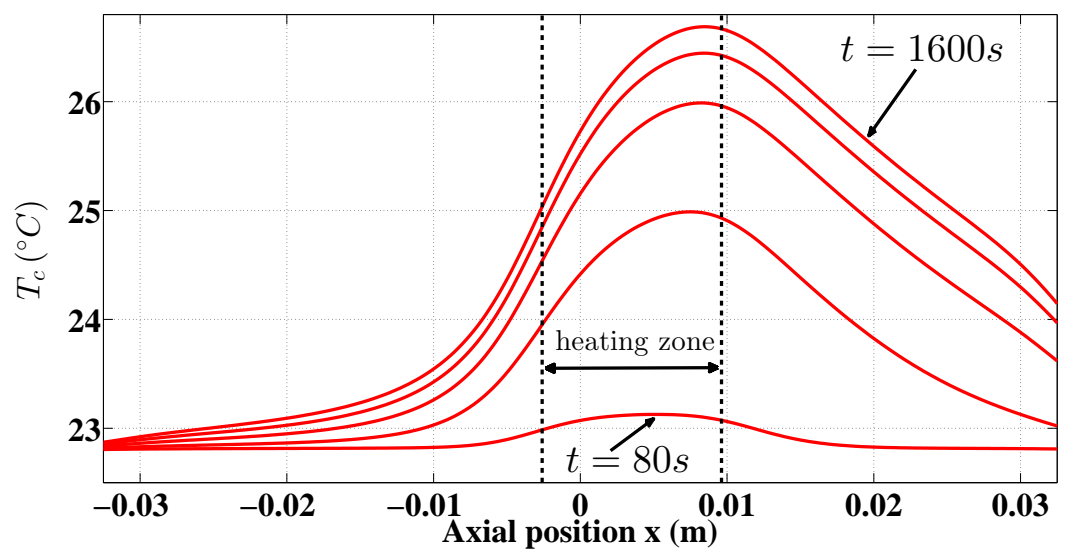

FIGURE 17: Estimated cold rear face temperature $T_{c}$ profile.

We can see that the reconstruction of the front face temperature, see Figure 14, is very good. In Figure 15 to Figure 17, at short times, we can see the symmetry with respect to the heating interval, with a downstream shift for longer times because of advection. One can notice the start of a steep decrease of the temperature distributions, for the longer times, in the downstream regions : this is due to the change of thickness of the front and rear walls in this region. In the downstream region, we can also see that we 
have $T_{b}>T_{w h}$ and $T_{b}>T_{w c}$ past the heating stop : this can be explained by the fact that the thermal diffusivity of fluid $a_{f}$ is slightly higher than that of walls $a_{s_{i}}$ and that $P e>1$.

As shown in section 4 , the surface heat source can be estimated by the technique proposed in this paper. In Figure 18 the estimated heat source is presented. In this experience, the surface heat source has been imposed electrically as a step function in time : the delay between the times $t=80 \mathrm{~s}$ and $t=1600 \mathrm{~s}$ can be explained by the thermal inertia of the heating system. The maximum is close to $500 \mathrm{~W} \cdot \mathrm{m}^{-2}$ for the last time shown $(1600 \mathrm{~s}$ "steady state"), with an average value over the heating interval lower than $400 W \cdot m^{-2}$ : it is coherent with the measured electrical power density of $405 \mathrm{~W} . \mathrm{m}^{-2}$ over the same time interval. The difference stems from the heat losses towards the insulating material at the back of the heating resistance and from the heat losses of the lateral surface of the copper bar, which are not taken into account in the model.

So, the source $q$ that is estimated here and shown in Figure 18 is just an equivanent one. Hence, it is interested to estimate also the flux $\varphi_{h}$ entering the external face of the hot face wall, see Figure 19, and compare it to its counterpart shown in Figure 9 and estimated from simulated hot face temperature measurements. One can notice in Figure 9 larger oscillations near the boundaries of the channel than in Figure 19, where the signal over noise ratios were about the same in the simulated and real experiments. This difference stems from the abrupt variation of the source of $\varphi_{h}$ in the simulated experiement (a Heaviside function for the source $q$ ) for short times, while the prsence of the heating device shown in Figure 12, which is not modelled, allowed a continuous variation of flux $\varphi_{h}$ in the real experiment, with a lot easier regularization that prevented large Gibbs phenomena at the two boundaries of the channel.

Other interesting information that can be derived are space distributions of the internal heat fluxes $\varphi_{w h}$ and $\varphi_{w c}$. In Figure 20, the estimated hot interface heat flux $\varphi_{w h}$ is presented. It is interesting to note that the heated fluid gives heat back to the wall past the heating region (negative flux). In Figure 21 the estimated cold interface heat flux $\varphi_{w c}$ is presented. Another non obvious effect can be found in this figure : the fluid upstream the heating region is colder than the cold wall, which means that this rear wall gives heat 
to the fluid (negative flux). The external cold heat flux, $\varphi_{c}$ is not shown here : it is proportional to the temperature variation of the cold face whose estimate is shown in Figure 17, which can be calculated as : $\varphi_{c}(x, t)=-h_{2}\left(T_{c}(x, t)-\right.$ $T_{\infty}$ ), (see equation 4 ).

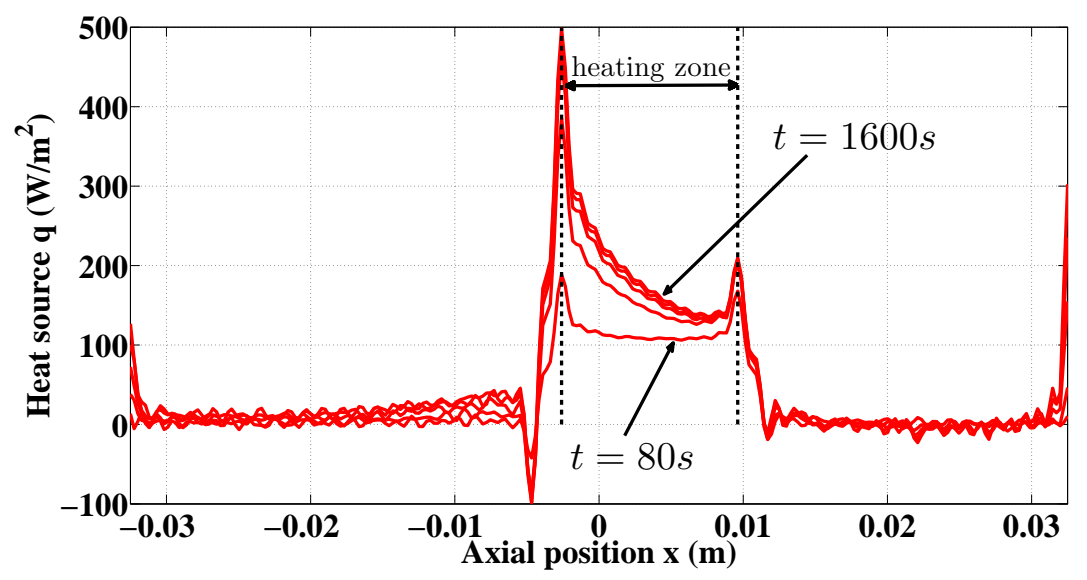

Figure 18: Estimated surface heat source $q$ (deconvolution, $\alpha_{t}=599$ for $\left.N_{t}=600\right)$ profiles.

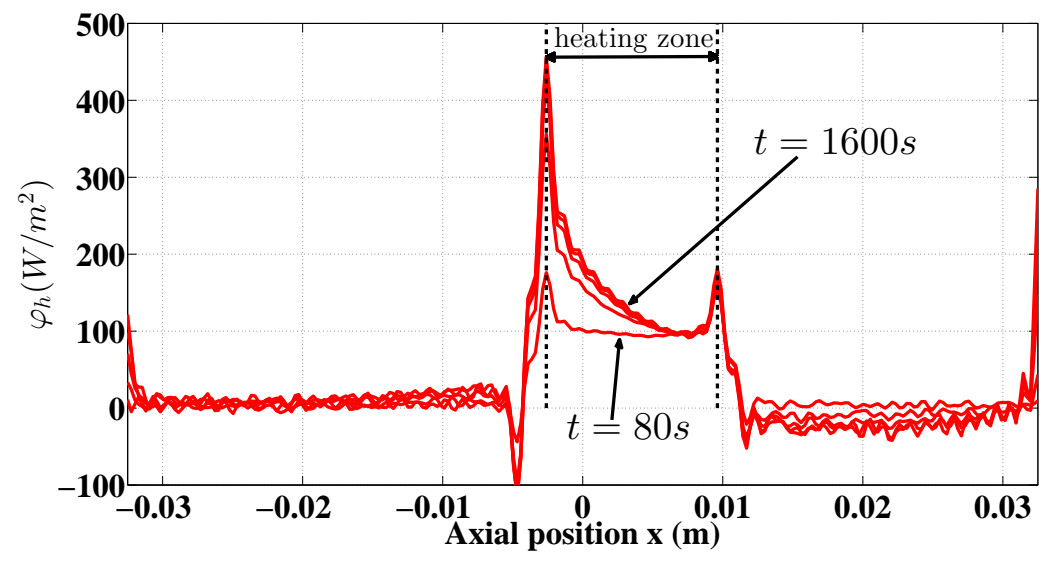

Figure 19: Estimated front face heat flux $\varphi_{h}$ profiles. 


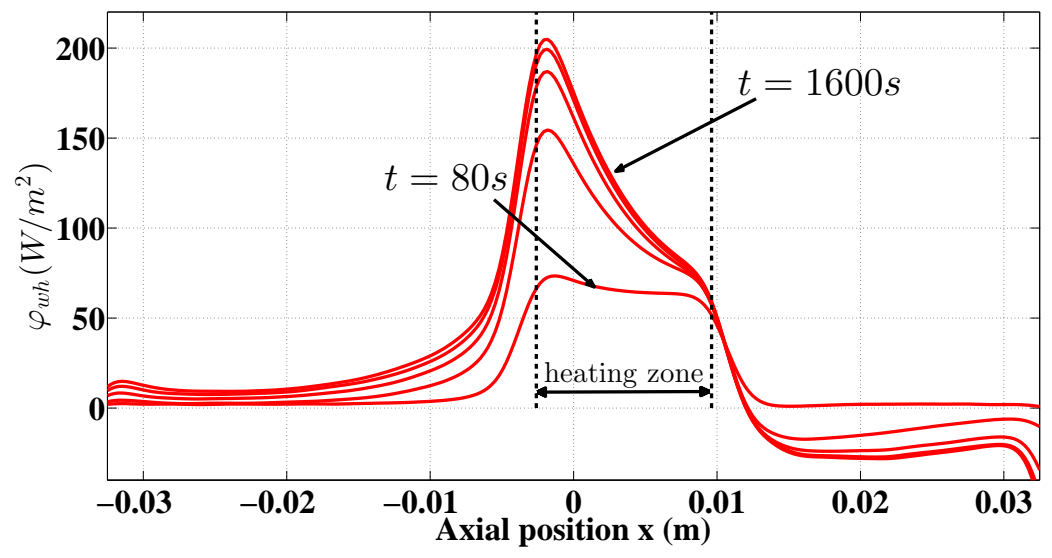

FIGURE 20: Estimated hot interface heat flux $\varphi_{w h}$ profiles.

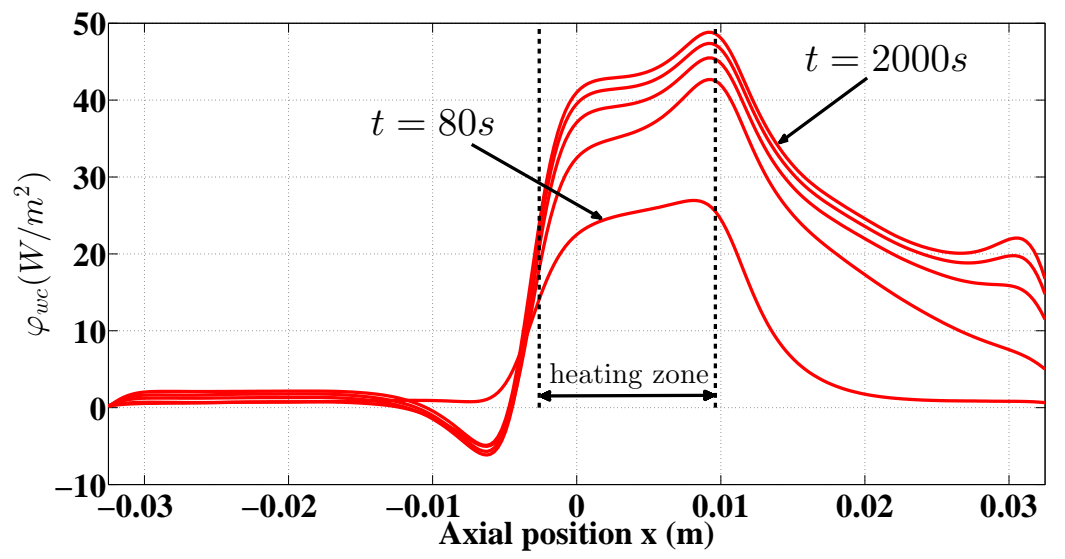

FigURE 21: Estimated cold interface heat flux $\varphi_{w c}$ profiles.

\section{Conclusion}

We have shown that in a mini heat extractor the heat source, as well as the internal normal heat flux and temperature distributions, including the bulk temperature of the liquid flow, can be estimated at any time from external mesurements using a non intrusive inverse technique and a semi-analytical model. This stems from the existence of analytical transfer functions in the double transformed domain, that is Laplace in time and Fourier in space, 
in the direction $x$ of the flow. This has also required the implementation of a numerical Laplace inversion algorithm, to make convolution products in time appear between Fourier harmonics of instantaneous $x$ distributions of the different quantities.

As in our previous work [2] where an instantaneous temperature response, at a given point of a channel, to an upstream thermal power excitation in the fluid, was explained by an impedance $Z$ involved in a convolution product, such can also be the case here. For example, the corresponding relationship between the intensity $Q(t)$ of the heating power and its point response at a point $x$ of the cold face is given by :

$$
\theta^{c}(x, t)=Z^{c}(x, t) * Q(t)
$$

where

$$
Z^{c}(x, t)=\frac{1}{2 L} \sum_{n=-n_{h}+1}^{n_{h}} \tilde{Z}_{n}^{c}(t) e^{\iota \alpha_{n} x} \quad \text { with } \quad \tilde{Z}_{n}^{c}(t)=L^{-1}\left[\frac{1}{\mathcal{C}_{n}(p)}\right]
$$

These analytical functions are available here because of the simple geometry of the channel that makes the use of the Quadrupole method possible. For a complexe system (ex : heat exchanger with fins, turbulent, rough walls, ...), it can be identified by a calibration experiment [2]. Let us also note, that transfer functions, once known (model reduction or experiemental identification) are alternatives to the classical detailed modelling which sometimes requires large calculation times and memory, especially in transient simulation. Let us remark here that in this work, the input of the inverse problem is the measured instantaneous temperature distribution over the front face, that is the face that is heated. This implies of course the implementation of some kind of space regularization to recover its Fourier spectrum and some time regularization to estimate the history of each harmonics of any of the quantities (temperture, heat fluxes or heat sources, see left column of Table 1) that are looked for. However, we are in a favorable configuration because the front face temperature measurements is characterized by the presence of high space frequencies, which explains the low levels of the estimation errors shown in Figure 4 to Figure 8 . Had the rear face temperature been measured, instead of the front one, the corresponding estimations would not have been as good, because of the necessary inversion of a temperature signal where 
high frequencies of the front face excitation have been damped by the solid walls and flowing fluid (low pass filter).

\section{Acknowledgment}

We thank Jamal Ouhajjou and Franck Demeurie for their involvement in the realization and optimization of the experimental setup.

\section{References}

[1] W. Al Hadad, D. Maillet, and Y. Jannot, "Modeling unsteady diffusive and advective heat transfer for linear dynamical systems : A transfer function approach," International Journal of Heat and Mass Transfer, vol. 115, pp. 304-313, 2017.

[2] W. Al Hadad, D. Maillet, and Y. Jannot, "Experimental transfer functions identification : Thermal impedance and transmittance in a channel heated by an upstream unsteady volumetric heat source," International Journal of Heat and Mass Transfer, vol. 116, pp. 931-939, 2018.

[3] J. V. Beck, B. Blackwell, and C. R. S. Clair Jr, Inverse heat conduction : Ill-posed problems. Wiley-Interscience, 1985.

[4] I. Tougri, M. Colaço, F. Bozzoli, and L. Cattani, "Internal heat transfer coefficient estimation in three-dimensional ducts through the reciprocity functional approach-an analytical approach and validation with experimental data," International Journal of Heat and Mass Transfer, vol. 122, pp. 587-601, 2018.

[5] Y. Jarny, D. Maillet, and D. Petit, "Inverse methods applied to forced convection in heat transfer, (in french : Méthodes inverses appliquées à la convection forcée en thermique)," Techniques de l'Ingénieur, Paris, p. 35, 2015.

[6] R. Raghunath, "Determining entrance conditions from downstream measurements," International communications in heat and mass transfer, vol. 20, no. 2, pp. 173-183, 1993.

[7] J. Bokar and M. Özisik, "An inverse analysis for estimating the timevarying inlet temperature in laminar flow inside a parallel plate duct," International journal of heat and mass transfer, vol. 38, no. 1, pp. 39-45, 1995. 
[8] F. Liu and M. Ozisik, "Inverse analysis of transient turbulent forced convection inside parallelplate ducts," International journal of heat and mass transfer, vol. 39, no. 12, pp. 2615-2618, 1996.

[9] H.-Y. Li and W.-M. Yan, "Inverse convection problem for determining wall heat flux in annular duct flow," Journal of heat transfer, vol. 122, no. 3, pp. 460-464, 2000.

[10] D. T. Lin, W.-M. Yan, and H.-Y. Li, "Inverse problem of unsteady conjugated forced convection in parallel plate channels," International Journal of Heat and Mass Transfer, vol. 51, no. 5-6, pp. 993-1002, 2008.

[11] H. Herwig and O. Hausner, "Critical view on "new results in microfluid mechanics" : an example," International Journal of Heat and Mass Transfer, vol. 46, no. 5, pp. 935-937, 2003.

[12] I. Perry, Y. Jannot, D. Maillet, and B. Fiers, "Effect of velocity distribution on external wall temperature field for a flat microchannel," Experimental Heat Transfer, vol. 23, no. 1, pp. 27-43, 2009.

[13] A. Degiovanni and B. Remy, "An alternative to heat transfer coefficient : A relevant model of heat transfer between a developed fluid flow and a non-isothermal wall in the transient regime," International Journal of Thermal Sciences, vol. 102, pp. 62-77, 2016.

[14] M. Vera and A. E. Quintero, "On the role of axial wall conduction in mini/micro counterflow heat exchangers," International Journal of Heat and Mass Transfer, vol. 116, pp. 840-857, 2018.

[15] D. Maillet, S. André, J.-C. Batsale, A. Degiovanni, and C. Moyne, Thermal quadrupoles: solving the heat equation through integral transforms. John Wiley \& Sons, 2000.

[16] R. C. Aster, B. Borchers, and C. H. Thurber, Parameter estimation and inverse problems. Elsevier Academic Press, 2012.

[17] P. C. Hansen, "Truncated singular value decomposition solutions to discrete ill-posed problems with ill-determined numerical rank," SIAM Journal on Scientific and Statistical Computing, vol. 11, no. 3, pp. 503518, 1990. 
[18] W. Al Hadad, D. Maillet, S. André, and B. Rémy, "Regularization using truncated singular value decomposition for estimating the fourier spectrum of a noised space distribution over an extended support," Computer Assisted Methods in Engineering and Science, vol. 21, no. 3-4, pp. 211222,2014 .

[19] Y. Rouizi, D. Maillet, and Y. Jannot, "Fluid temperature distribution inside a flat mini-channel : semi-analytical wall transfer functions and estimation from temperatures of external faces," International Journal of Heat and Mass Transfer, vol. 64, pp. 331-342, 2013.

[20] Y. Rouizi, W. Al Hadad, D. Maillet, and Y. Jannot, "Experimental assessment of the fluid bulk temperature profile in a mini channel through inversion of external surface temperature measurements," International Journal of Heat and Mass Transfer, vol. 83, pp. 522-535, 2015.

[21] P. C. Hansen, "Analysis of discrete ill-posed problems by means of the l-curve," SIAM review, vol. 34, no. 4, pp. 561-580, 1992.

[22] H. Stehfest, "Algorithm 368 : Numerical inversion of laplace transforms [d5]," Communications of the ACM, vol. 13, no. 1, pp. 47-49, 1970.

[23] H. Stehfest, "Remark on algorithm 368 : Numerical inversion of laplace transforms," Communications of the ACM, vol. 13, no. 10, p. 624, 1970.

[24] COMSOL Multiphysics version 3.4. 